\title{
Effect of particle size on the measurement of the apparent contact angle in sand of varying wettability under air-dried conditions
}

\author{
Yunesh Saulick $^{1, \text { a }}$, Sergio Lourenço ${ }^{1}$ and Béatrice Baudet ${ }^{1}$ \\ ${ }^{1}$ The University of Hong Kong (HKU), Pokfulam, Hong Kong, Hong Kong SAR, China
}

\begin{abstract}
Changes in the wettability of soil are known to affect several processes such as infiltration and the shear strength of soil. In this study, the wettability of a medium to fine sand was chemically modified by using different concentrations of dimethyldichlorosilane (DMDCS). The sessile drop method (SDM) was used for the assessment of wettability of hydrophobised Leighton Buzzard Sand (LBS). The results demonstrate that beyond a concentration of $2 \mathrm{~g}$ per $\mathrm{kg}$ of LBS, the finer fraction had its apparent contact angle (ACA) increased up to $115^{\circ}$ while the maximum ACA attained by the coarser fractions was $100^{\circ}$. At such high concentration of DMDCS, the effect of trapped air, which is known to increase the ACA, was found to be either small or insignificant. The standard deviations of the ACAs agreed well with past studies. The most important factors contributing to the water-repellent behaviour of chemically synthesised sand were attributed to the characteristics of the particles; these include surface area and particle shape.
\end{abstract}

\section{Introduction}

The wettability of natural soils is known to alter significantly with time due to numerous reasons such as organic matter and soil texture [1, 2, 3]. For instance, a decrease in particle size due to crushing will cause a significant increase in the surface area of the particles, which will in turn require more organic matter for coating. A decrease in the wettability of soils causes increased surface runoff and enhance preferential flow in soils [3-4]. Several techniques to assess the wettability of soils have been established throughout the literature; they include direct methods of measurement of the apparent contact angle (ACA) such as the sessile drop method (SDM) and indirect methods such as the capillary rise methods.

In the field of geotechnical engineering, especially where unsaturated soils are involved, the wetting characteristic of soils is important because they have a significant influence on suction. Lourenço et al. [5] demonstrated that for a mixture of sand and clay, its ability to retain water decreased as the ACA approached $90^{\circ}$. Shear strength of non-wettable soils as evaluated by direct shear tests performed by Bardet et al. [6] using soils coated by wax demonstrated that there was no change in the angle of shear resistance compared to wettable samples. However, Lee et al. [7] showed that a decrease in the peak friction angle (tests carried out with samples having same initial void ratios) could be observed when their samples were made less wettable by means of a silane coating.

\footnotetext{
a Corresponding author: yunesh@connect.hku.hk
}

The influence of wettability on the behaviour of unsaturated soils is relevant in studies of porous and granular materials down to the grain-size level. Here, we investigate the effect of the grain-size on the wettability of a medium to fine commercial sand made repellent to different extents by means of an organosilicon compound.

\section{Materials and methods}

\subsection{Soil material}

The soil used in the study was Leighton Buzzard Sand (LBS), which originates from quarries in southern UK. Due to its rich content in silica (mainly in the form of quartz comprising nearly $98 \%$ of the sand), LBS exhibits excellent properties such as chemical inertness. The LBS sample was sieved by means of a mechanical shaker to obtain four sieve fractions namely 63-212, 212-300, 300425 and $425-600 \mu \mathrm{m}$. The samples were initially airdried, the water content of which were between 1 and $2 \%$. Table 1 shows the particle shape characteristics extracted from a dynamic shape analyser (QICPIC; Sympatec GmbH, Clausthal-Zellerfeld, Germany). For each of the aforementioned sieve sizes, a minimum of 10,000 images was analysed. From the shape parameters extracted, a general increase in sphericity, aspect ratio and convexity was observed as the size of the particles increased. The sphericity as defined by the QICPIC is the ratio of the equivalent perfect circle to the actual perimeter for a given area. The value of the aspect ratio is 
given by the ratio of of the minimum to maximum Feret diameters while convexity is calculated as a ratio of the actual to the convex area. The convex area refers to the circumscribed borderline to the 2-D outline of the particle.

Table 1. Particle shape characteristics of the different sieve fractions obtained from a dynamic shape analyser.

\begin{tabular}{|c|c|c|c|c|}
\hline $\begin{array}{c}\text { Particle } \\
\text { attributes }\end{array}$ & $\begin{array}{c}\mathbf{6 3 - 2 1 2} \\
\boldsymbol{\mu m}\end{array}$ & $\begin{array}{c}\mathbf{2 1 2 - 3 0 0} \\
\boldsymbol{\mu m}\end{array}$ & $\begin{array}{c}\mathbf{3 0 0 - 4 2 5} \\
\boldsymbol{\mu m}\end{array}$ & $\begin{array}{c}\mathbf{4 2 5 - 6 0 0} \\
\boldsymbol{\mu m}\end{array}$ \\
\hline Sphericity & 0.8806 & 0.8884 & 0.8926 & 0.8942 \\
\hline Aspect Ratio & 0.7294 & 0.7454 & 0.7611 & 0.7599 \\
\hline Convexity & 0.9200 & 0.9381 & 0.9487 & 0.9578 \\
\hline
\end{tabular}

\subsection{Hydrophobization reaction}

To a $40 \mathrm{~g}$ sample of LBS comprising of an equal proportion of the aforementioned sieve fractions, was added an incremental amount of Dimethyldichlorosilane (molecular weight of $129.06 \&$ density $1.06 \mathrm{gcm}^{-3}$ from Acros Organics, New Jersey, USA) drop by drop from a single channel pipette (Eppendorf Research ${ }^{\circledR}$ plus) with constant and gentle stirring. The end of the coating reaction between the sand and the chemical solution was indicated by the formation of hydrogen chloride fumes. The next steps were to leave the samples in the fume hood for half an hour and then keeping them in Ziploc bags for 24 hours prior to any measurements.

\subsection{Apparent contact angle (ACA) measurement}

The ACAs were determined by a goniometer (Drop Shape Analyser 25 from KRÜSS $\mathrm{GmbH}$ ) where the motion of a $10-\mu 1$ drop of de-ionised water was recorded by a 1394 AVT CCD camera upon contact on a 76 x 26 x $1 \mathrm{~mm}$ slide to which the soil sample was pasted via a a double-sided tape following the method proposed by Bachmann et al. [8]. Similarly to Shang et al. [9] in their study of porous materials, the initial ACA was considered the most representative measure of wettability. The initial ACA was taken from the first stable frame from the movie (approximately $50 \mathrm{~ms}$ after contact with the substrate) as the contact angle hysteresis due to volume loss is least likely to occur at this stage. The magnitude of the ACAs was obtained by developing a semi-automated method combining the low-bond axisymmetric drop shape analysis (LBADSA) method developed by Stalder et al. [10] and a manual adjustment of the baseline. All ACAs measurements were performed at a temperature of $23 \pm 1{ }^{\circ} \mathrm{C}$ and relative humidity of $55 \pm 5 \%$.

\section{Results and discussions}

The relationship between the different size fractions and the ACAs is presented in Figure 1. The standard deviations of the ACAs were at most $\pm 6^{\circ}$ and in line with the studies of Bachmann et al. [11]. It can be seen that at relatively small concentrations of DMDCS, the change in ACAs within the different size fractions is small although the lowest ACAs are usually associated with the finer materials. As the amount of DMDCS increases, the difference between the sizes becomes more apparent as indicated by the steeper slopes. A possible justification for such behaviour is that the smaller size fractions require a larger amount of DMDCS to be coated due to their larger surface areas [12]. The overlapping lines at the two highest concentrations, which were investigated (Figure 1), are probably due to the difficulty in extracting the values of the ACAs. This arises when adjusting the baseline and the optics settings on the image output from the Drop Shape Analyser 25 especially at high ACAs.

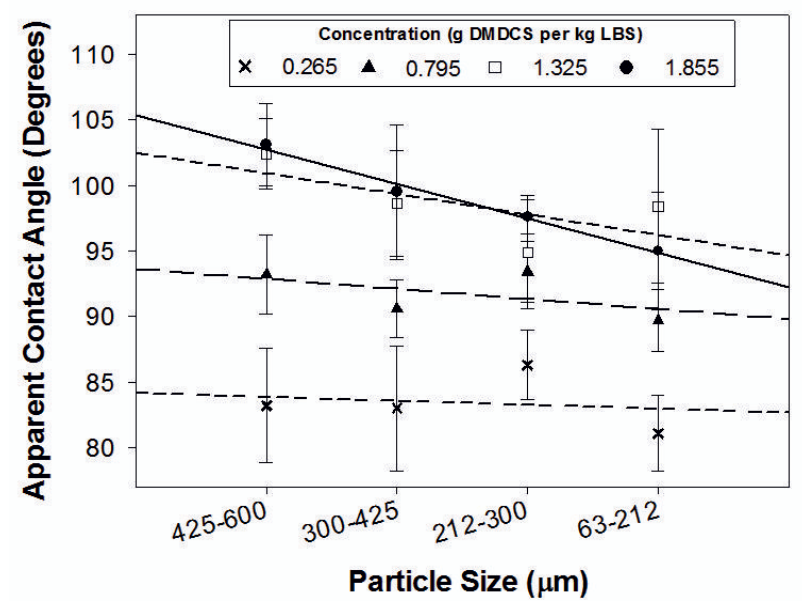

Figure 1. Apparent contact angles as a function of particle size in samples with different concentration of DMDCS.

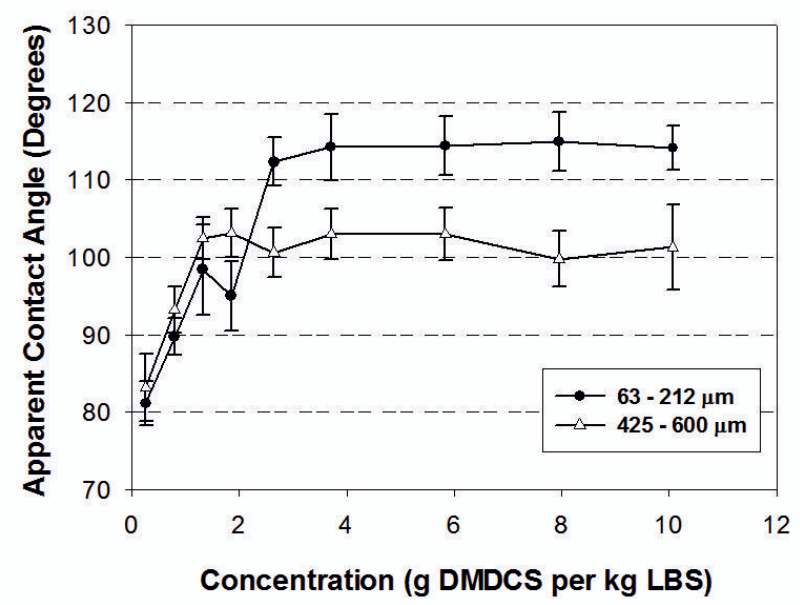

Figure 2. Maximum apparent contact angles attained by the coarsest and finest fractions.

To determine the percentage of particles coated, the maximum ACA by the different fractions was first identified. The results are shown in Table 2 and Figure 2 illustrates the plot for the 63-212 $\mu \mathrm{m}$ and 425-600 $\mu \mathrm{m}$ sieve fractions. From Figure 2, it can be seen that for a concentration of DMDCS of up to $2 \mathrm{~g}$ per $\mathrm{kg}$ of LBS, it is 
unclear which of the two fractions show the larger ACA due to the overlapping error bars. However, as the concentration increases beyond this, the maximum ACA attained by the $300-425$ and $425-600 \mu \mathrm{m}$ sieve fractions is $105.5 \pm 5.38^{\circ}$ while a maximum value of $116.6 \pm 2.86^{\circ}$ was reached for the 63-212 and 212-300 $\mu \mathrm{m}$ sieve fractions. In addition, the difference in the maximum ACAs between the two sieve-fractions in Figure 2 demonstrates that the assumption of a larger fraction of air being trapped in the coarser particles (which usually enhances ACAs) compared to the finer ones is not justified for the relative higher ACA in fine sand.

Table 2. ACA with increasing concentration of DMDCS.

\begin{tabular}{|c|c|c|c|c|}
\hline & \multicolumn{4}{|c|}{ ACA (Degrees) } \\
\hline $\begin{array}{c}\text { Concent } \\
\text { ration } \\
\mathbf{( g} \\
\text { DMDCS } \\
\text { / kg of } \\
\text { Soil) }\end{array}$ & $\mathbf{6 3 - 2 1 2} \boldsymbol{\mu \mathbf { m }}$ & $\mathbf{2 1 2 - 3 0 0} \boldsymbol{\mu \mathbf { m }}$ & $\mathbf{3 0 0 - 4 2 5} \boldsymbol{\mu \mathbf { m }}$ & $\mathbf{4 2 5 - 6 0 0} \boldsymbol{\mu \mathbf { m }}$ \\
\hline 0.265 & $81.1 \pm 2.86$ & $86.3 \pm 2.62$ & $83.0 \pm 4.78$ & $83.2 \pm 4.34$ \\
\hline 0.795 & $89.7 \pm 2.37$ & $93.4 \pm 2.34$ & $90.6 \pm 2.21$ & $93.2 \pm 3.03$ \\
\hline 1.325 & $98.4 \pm 5.86$ & $94.9 \pm 4.35$ & $98.6 \pm 4.02$ & $102.4 \pm 2.71$ \\
\hline 1.855 & $95.0 \pm 4.51$ & $97.6 \pm 1.33$ & $99.5 \pm 5.13$ & $103.1 \pm 3.15$ \\
\hline 2.650 & $112.3 \pm 3.12$ & $100.4 \pm 1.74$ & $102.0 \pm 4.14$ & $100.6 \pm 3.21$ \\
\hline 3.710 & $114.2 \pm 4.26$ & $111.7 \pm 4.18$ & $92.4 \pm 3.73$ & $103.0 \pm 3.24$ \\
\hline 5.830 & $114.4 \pm 3.77$ & $110.8 \pm 4.98$ & $103.7 \pm 4.00$ & $103.0 \pm 3.45$ \\
\hline 7.950 & $114.9 \pm 3.80$ & $116.6 \pm 2.86$ & $103.4 \pm 4.07$ & $99.8 \pm 3.54$ \\
\hline 10.070 & $114.1 \pm 2.84$ & $113.9 \pm 2.28$ & $105.5 \pm 5.38$ & $101.3 \pm 5.44$ \\
\hline
\end{tabular}

The fully methylated surfaces of the coarse and fine sand samples were taken as $100^{\circ}$ and $115^{\circ}$ respectively. Using these values and the Cassie equation (Eq. 1), the percentage of sand coated within each of the four sieve fractions for concentration up to $1.855 \mathrm{~g} \mathrm{~kg}^{-1}$ has been calculated and illustrated in Figure 3. The larger shape parameters associated with the coarser sieve fractions showed a higher tendency to coat by the DMDCS at and below this value.

$$
\cos A C A=f_{1} \cos \theta_{1}+f_{2} \cos \theta_{2}
$$

In Eq. 1, $f_{1}$ and $f_{2}$ represent the fraction of soil having intrinsic contact angle $\theta_{1}$ and $\theta_{2}$ respectively. For the unmethylated soil fraction, $\theta_{1}$ was taken as $0^{\circ}$ and $\theta_{2}$ denotes the fully methylated soil surface.

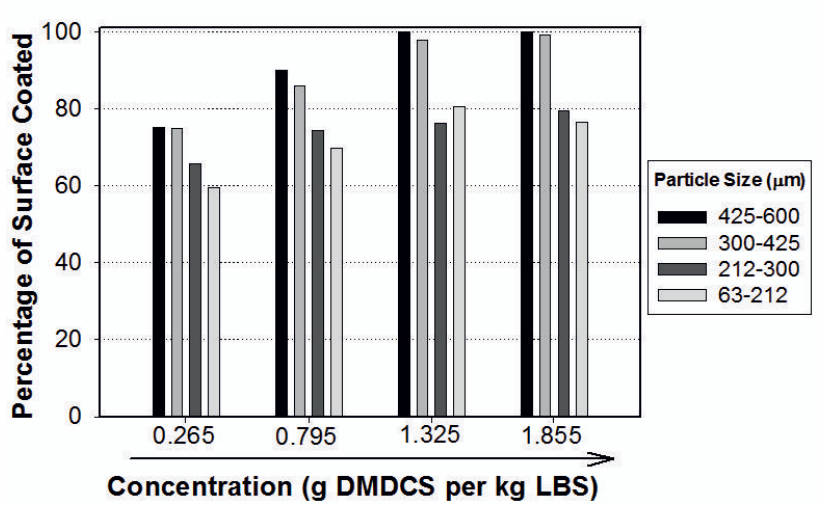

Figure 3. Percentage of surface coated within each sieve fraction.

\section{Conclusions}

This study shows that particle size is a significant parameter to be considered if the wetting properties of soils are to be exploited in the field of geotechnical engineering. It was found that the chemically synthesised water repellent soils examined were more water repellent with the finer fractions for a concentration above $2 \mathrm{~g}$ DMDCS per $\mathrm{kg}$ of LBS. Below this value, it is not distinguishable which of the two sieve sizes had a larger ACA. Furthermore, the influence of particle size on the wettability of chemically synthesised sand seem to suggest that there are threshold values regarding the particle size and DMDCS concentration which dictate how and to which extent the ACA will vary.

The maximum ACA values reached by the coarser and finer fractions were around $100^{\circ}$ and $115^{\circ}$ respectively. At high concentration of DMDCS, it was concluded that the effect of trapped air within soil particles is small as compared to the particle characteristics such as their surface area and shape.

\section{Acknowledgement}

This research work has been possible through the funding support provided by The University of Hong Kong. Help and comments by Mr. Shuang Zheng as well as laboratory support from Mr. N.C. Poon are much appreciated.

\section{References}

1. R.D. Bond, Trans. 9th Int. Congr. Soil Sci. 1, 339-347 (1968)

2. L.F. DeBano, L.D. Mann, D.A. Hamilton, SOIL SCI. SOC. AMER. PROC. 34, (1970)

3. S. Doerr, R. Shakesby, R. Walsh, Earth Sci. Rev. 51, 33-65 (2000)

4. C. Ritsema, L. Dekker, Aust. J. Soil Res. 34, 475-487 (1996)

5. S.D.N. Lourenço, N.C. Jones, S.H. Doerr, R. Bryant, Vadose Zone J. 14, (2015)

6. J.P. Bardet, J. Jesmani, N. Jabbari, EJGE. 16, 451-461 (2011) 
7. C. Lee, H. Yang, T.S. Yun, Y. Choi, S. Yang, Vadose Zone J. 14, (2015)

8. J. Bachmann, A. Ellies, K.H. Hartge, J HYDROL. 231-232, 66-75 (2000)

9. J. Shang, M. Flury, M. Harsh, J.B. Harsh, R.L. Zollars, J COLLOID INTERF SCI. 328, 299-307 (2008)

10. A.F. Stalder, T. Melchior, M. Muller, D. Sage, T. Blu, M. Unser, COLLOID SURFACE A. 364, 72-81 (2010)
11. J. Bachmann, S.K. Woche, M.-O. Goebel, WATER RESOUR RES. 39, 1353-1366 (2003)

12. I.E. Dubois, S. Holgersson, S. Allard, M.E. Malmström, PROC MONOGR ENG WATE. 717-720 (2010) 